\title{
Transvascular Growth of Hepatocellular Carcinoma
}

\author{
AR Muneer ${ }^{1}$, S Biji ${ }^{2}$, OS Suman ${ }^{1}$, KJ Peter ${ }^{1}$, G Vijayaraghavan $^{1}$, D Leena ${ }^{3}$ \\ Departments of ${ }^{1}$ Cardiology and ${ }^{3}$ Pathology, Kerala Institute of Medical Sciences, Trivandrum, Kerala; ${ }^{2}$ Department of Cardiology, \\ SUT Royal Hospital, Trivandrum, Kerala, India.
}

\section{Corresponding Author:}

Dr. Abdul Rahaman Muneer

Email: dr.reenum@yahoo.com

This is an Open Access article distributed under the terms of the Creative Commons Attribution License (creativecommons.org/ licenses/by/3.0).

Received

Accepted

Published
January 24, 2017

March 21, 2017

April 10, 2017

\begin{abstract}
Background: Cardiac tumors may be primary (benign or malignant) or metastatic (malignant). They can cause inflow-outflow tract obstruction, thromboembolism, arrhythmias, or pericardial disorders. Case Report: A 30 year old male presented with cough and dyspnea of one month duration. Routine echocardiography revealed a large tumor mass filling the entire right atrium. Computed tomography delineated tumor arising from the left lobe of the liver and entering through the inferior vena cava (IVC) to right atrium. Ultrasound guided biopsy demonstrated hepatocellular carcinoma. The patient initially improved with oral sorafenib (receptor tyrosine kinase inhibitor) and then succumbed to his primary illness after six months. Conclusion: We report here transvascular growth of hepatocellular carcinoma through IVC to right atrium, a rare phenomenon.
\end{abstract}

Keywords: Echocardiography, Heart Neoplasms, Liver Neoplasms, Thromboembolism, Vena Cava.

\section{Introduction}

Tumors occurring in the heart are rare [1]. Tumor extensions through vascular pathways are uncommon phenomena [1]. Hepatocellular carcinoma patients with tumor extended into the right atrium (RA) are rare, and very few have been reported [2]. We report here a rare event of transvascular growth of hepatocellular carcinoma.

\section{Case Report}

A 30 year old gentleman presented with cough and effort dyspnea of one month duration. Clinical examination revealed a markedly elevated jugular venous pulse (JVP) with prominent "a" and " $\mathrm{j}$ " waves. There was no cardiomegaly and there were no signs of cardiac failure. Abdomen was distended with enlarged liver which appeared firm and extended beyond midline. Patient was HBsAg positive with deranged liver function. Alpha feto-protein $(>50,000 \quad \mathrm{IU} / \mathrm{mL})$ was suggestive of hepatocellular carcinoma. The 12 lead electrocardiogram was normal.
The two dimensional transthoracic echocardiogram in the parasternal short axis view at the level of great arteries demonstrated a large mass extending from the lumen of the inferior vena cava into the right atrium and filling the medial half of the right atrium [Fig.1a]. The apical four chamber view offered a much better image of the tumor mass [Fig.1b]. It was evident that the tumor mass was not attached either to the atrial septum or right atrial wall and the tip of the mass was projecting just beyond the tricuspid annulus in diastole. Intact tricuspid valve could be demonstrated. The mass did not show any independent mobility. Color flow showed an obstruction of flow across the tricuspid valve with a peak gradient varying from 5 to 12 mmHg [Fig.1c,d].

The sub-costal view imaged the inferior vena cava clearly except its opening into the right atrium. The right atrial opening was filled with the tumor mass [Fig.2a]. It was interesting to note that the tumor mass was an extension from the liver and it had ruptured the liver capsule and extended into 
the terminal inferior vena cava and right atrium. Computed tomography (CT) abdomen [Fig.2b] in coronal view revealed a large ill defined, heterogeneous, predominantly iso-dense lesion, involving segment II, III, partly IVa of the left lobe of liver. The lesion was occluding middle, left hepatic veins and extended into infra-hepatic and supra-hepatic inferior vena cava (IVC) and bulged into the right atrial lumen where it measured $5.3 \times 4.9 \mathrm{~cm}$. Tru cut needle biopsy from liver lesion was done. The diagnosis was confirmed as hepatocellular carcinoma. The patient was put on oral sorafenib (receptor tyrosine kinase inhibitor). The patient initially improved and then succumbed to his primary illness after six months.

\section{Discussion}

The incidence of secondary cardiac tumors is variable and it ranges between $1.6 \%$ and $20.6 \%$. The most common sources of cardiac metastases include lung, breast and the lympho-reticular malignancies [3-5]. Secondary tumors spread to the heart through various mechanisms, like lymphatic or hematogenous pathways, local spread, or a transvenous extension. Retrograde spread through the lymphatic channels is the most common mode of spread [1]. Malignant melanoma has the highest rate of cardiac metastasis because of their greater predisposition for generalized hematogenous spread $[1,6]$.

Certain tumors such as renal cell carcinoma and hepatocellular carcinoma (HCC) can spread through an endovascular channel (transvenous) into the inferior vena cava and grow into the right atrium $[1,4,7,8]$. Cardiac metastasis is considered an unusual and rare presentation of $\mathrm{HCC}$ and is seen in about $2 \%$ of cases [4,9]. HCC metastases tend to spread mainly through lymphatic channels, hematogenous or direct infiltration, hematogenous extension being most common [9]. Hematogenous extension may result in hepatic, portal veins or vena cava involvement and right atrial intracavitary lesion, rarely causing obstruction [4]. Low
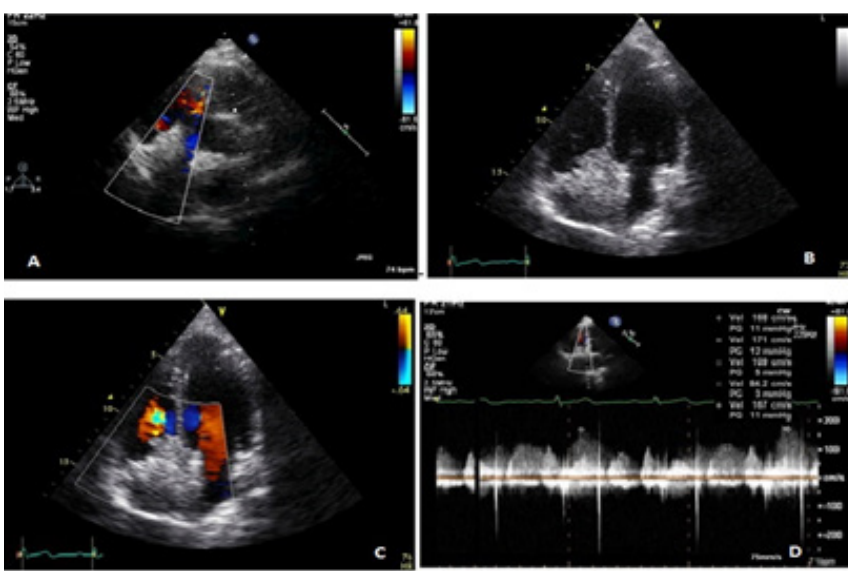

Fig.1: (Trans thoracic echocardiography views) (a): Short axis view at the level of great arteries demonstrating a large mass extending from the lumen of IVC into medial half of the $R A$. (b): Two dimensional transthoracic apical four chamber view showing the tumor mass filling up the medial aspect of the RA and free tricuspid valve leaflets. (c): Color flow across tricuspid valve in apical four chamber view showing an obstruction of flow across the tricuspid valve. (d): Doppler flow across tricuspid valve in apical four chamber view revealing the peak gradient varying from 5 to $12 \mathrm{mmHg}$ depending on the respiratory phase.
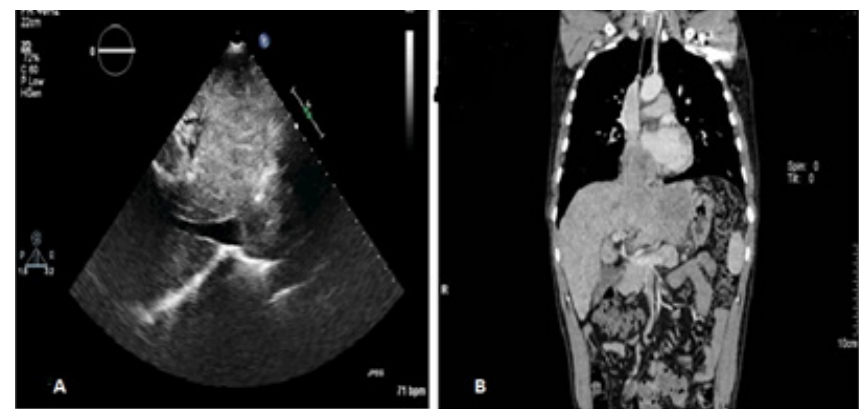

Fig.2(a): Sub-costal view in trans-thoracic echocardiography showing the IVC. RA opening is filled with the tumor mass and is an extension from the liver and it has ruptured the liver capsule and extending into the terminal IVC and RA. (b): CT scan of abdomen in coronal view revealing a large ill-defined iso-dense lesion, involving left lobe of liver. The lesion is also occluding middle, left hepatic veins and extending into infrahepatic and supra-hepatic IVC and bulging into right atrium.

intra-cavitary pressure, the slower blood flow and the lesser contractile force is thought to favor the seeding of cancer cells to the right heart chambers [4]. Continuous tumor thrombus extending from the hepatic vein, IVC and into the RA is also described, as was seen in our case [9]. 
Most cardiac metastases are clinically silent, since the clinical picture is usually dominated by generalized tumor spread $[1,4]$. Cardiac metastases commonly present with dyspnea, palpitations, lower limb edema, chest pain and atrial flutter or fibrillation and the right atrium is most commonly involved $[7,8,10]$. The initial investigation of choice to detect cardiac metastasis and their complication is two-dimensional echocardiography, as it is noninvasive, pain free and both pericardial involvement and intra-cavitary lesions can also be detected with high sensitivity $[1,11]$

Intra-cardiac metastases, even though rare, should also be included in the differential diagnosis, along with primary tumors of the heart, thrombus, vegetation, marantic endocarditis and foreign body like pacing leads [1]. Cardiac metastatic manifestations are a late occurrence in vast majority of the cases, by then, the primary tumor disease is in an advance stage with generalized tumor spread. Because of this, cardiac treatment is mostly palliative.

\section{Conclusion}

HCC bursting the liver capsule and extending into the terminal part of IVC and entering the right atrium is a very rare presentation.

Contributors: ARM, SB: Manuscript writing and literature review; OSS: Echocardiography and literature review; GV, KJP: Critical review of manuscript; DL: Histopathology and manuscript review. ARM will act as guarantor. All authors approved the final version of the manuscript.

Funding: None; Competing interests: None stated.

\section{References}

1. Reynen K, Köckeritz U, Strasser RH. Metastases to the heart. Annals of Oncology. 2004;15:375-381.

2. Luo X, Zhang B, Chen X. Hepatocellular carcinoma with tumor thrombus occupying the right atrium and portal vein. Medicine (Baltimore). 2015;94(34):e1049.

3. Rafajlovski S, Tatic V, Ilic S, Kanjuh V. Frequency of metastatic tumors in the heart. Vojnosanit Pregl. 2005;62:915-920.

4. Bussani R, F De-Giorgio, Abbate A, Silvestri F. Cardiac metastases. J Clin Pathol. 2007;60:27-34.

5. Smith C. Tumors of the heart. Arch Pathol Lab Med. 1986;110:371-374.

6. Glancy DL, Roberts WC. The heart in malignant melanoma. A study of 70 autopsy cases. Am J Cardiol.1968;21:555-571.

7. Goldberg AD, Blankstein R, Padera RF. Tumors metastatic to the heart. Circulation. 2013;128:17901794.

8. Yusuf SW, Bathina JD, Qureshi S, Kaynak HE, Banchs $\mathrm{J}$, Trent JC, et al. Cardiac tumors in a tertiary care cancer hospital: clinical features, echocardiographic findings, treatment and outcomes. Heart Int. 2012;7:e4.

9. Albackr HB. A large right atrial mass in a patient with hepatocellular carcinoma: Case report and literature review. J Saudi Heart Association. 2014;26:174-178.

10. Liu YC, Ho YL, Huang GT, Chen DS, Sheu JC, Chen $\mathrm{CH}$. Clinical manifestations and survival of patients with hepatocellular carcinoma and cardiac metastasis. J Gastroenterol Hepatol. 2010;25:150-155.

11. Alhakeem M, Arabi A, Arab A, Guerra RA. Unusual sites of metastatic involvement: intracardiac metastasis from laryngeal carcinoma. European J Echocardiography. 2008;9:323-325. 